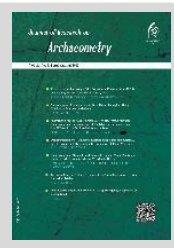

\title{
Gypsum Nails as a Solution for Efficient Strengthening and Maintaining of Historical Architectural Decorations
}

\author{
Yaser Hamzavi* \\ Assistant Professor, Faculty of Applied Arts, Tabriz Islamic Art University, Tabriz, IRAN
}

Received: 29/11/2017

Accepted: 22/12/2017

With regard to the dry climatic conditions of Iran, cob and gypsum mortars were used in architectural arrays of many regions of Iran, to create the scratch coat (Arriccio) and fine coat (Intonaco) in the historical periods. The thickness of the fine coat was often very low (one-two $\mathrm{mm}$ ). In this regard, one of the examples is the City of Yazd. One of the buildings made in the eighth century (Islamic calendar) with the mentioned properties in its scratch and fine coats in architectural arrays is the mausoleum of Rokn al-Din in Yazd (1946), which has several architectural arrays, such as: embossed gypsum, mold, painting inscriptions, decorative paintings, gilding arrays and tiling. The most important issue observed in the arrays of this type of buildings is when the straws and vegetable fibers found in cob are consumed by termites, which is recognized as one of the common damages to the buildings in the country center. This phenomenon leads to loss of coherence in the scratch coat, turning it into a weak powder. This damage to the scratch coat is associated with detachment of the underlying layers in the architectural array. There is an extremely thin layer of fine coat, which is completely detached from many parts of the underlying layer. In such conditions, other array holders are gypsum and gilding arrays and wall paintings. In general, it is significantly difficult to maintain this type of architectural arrays. One of the issues that makes restoration more difficult and limits the use of materials and methods to restore a building, is the white background of architectural arrays in these buildings, which makes it impossible to use any strengthening method in these buildings since it leads to the formation of a yellow and dark color on a white background. In addition, filling the empty space behind the gypsum fine coat (without considering the fact that this layer is significantly thin) leads to the separation and collapse of the fine coat and the implemented arrays on the layer (with regard to the gravitational force of the earth). The thin gypsum layer, which is similar to egg shell and is separated from the scratch coat, can be crushed with minimum pressure of the hand. Moreover, the significantly weak attachment of many mold gypsum arrays to the fine coat, the smallest collision, and vibration in that area leads to the separation and collapse of the decorative layer. To eliminate these problems, the soil in the empty space between the fine coat and support was cleaned at first, followed by fixing the upper and lower surface of the fine coat. The sample was created to select the proper material and method, at the end of which the following material and method were selected: fixing the surface with the solution of $6 \%$ Acryloid B-72 in ethanol and injecting the mentioned solution to the back of the fine coat. In the next stage, a cavity with the depth of about eight $\mathrm{cm}$ was created in parts of the support that experienced breaking or collapsing (brick wall or ceiling). It should be noted that the diameter of the cavity was small on the surface of the support (about two $\mathrm{cm}$ ), and the more the depth increased, the more the cavity diameter was enlarged. The next phase involved the injection

*Corresponding author: y.hamzavi@tabriziau.ac.ir 
and implementation of gypsum mortar to the lower layers. To this end, the local gypsum of Yazd and eremurus powder were exploited. Injection of the mortar will take a relatively long time and will be carried out in several stages. In each stage, a small amount of mortar is injected into the back of the fine coat and inside the cavity made in the support layer. After each injection phase, two hours is required for complete hardening of gypsum and initiation of the next injection operation. The cavities, along with $10 \mathrm{~cm}$ from around the cavity and between the scratch and fine coats, are completely filled with gypsum, which results in the attachment of layers to each other. In addition, this mortar acts like a nail, attaching the gypsum fine coat to the lower layers. In this experiment, the described strengthening method was called gypsum nail. Implementation of gypsum nails with relatively specific distances from each other $(50-100 \mathrm{~cm})$ will lead to the fixing of the fine coat and layers attached to its surface without putting pressure on the thin gypsum fine coat. 


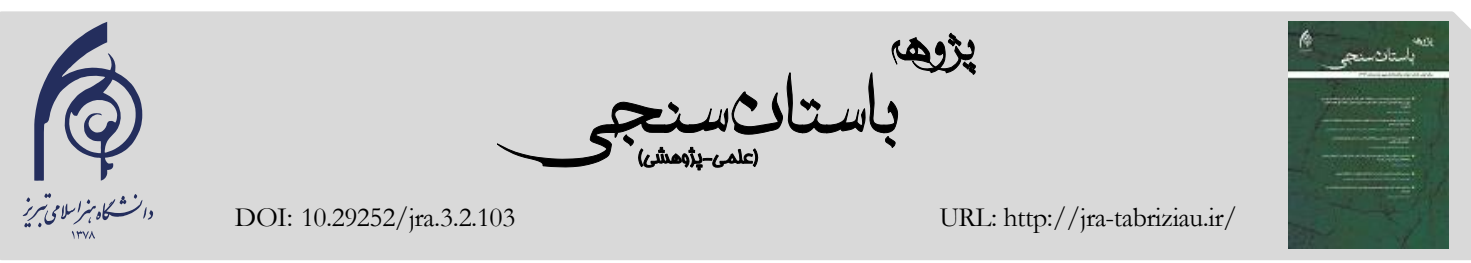

يادداشت فَنى

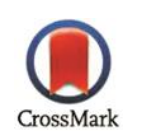

\title{
استفاده از ميخ تَّيى، راهكارى براى استحكامبخشى و

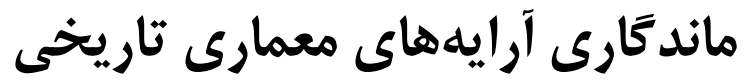

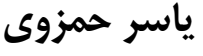 \\ استاديار، دانشكدة هنرهاى كاربردى، دانشكاه هنر اسلامى تبريز، تبريز، ايران
}

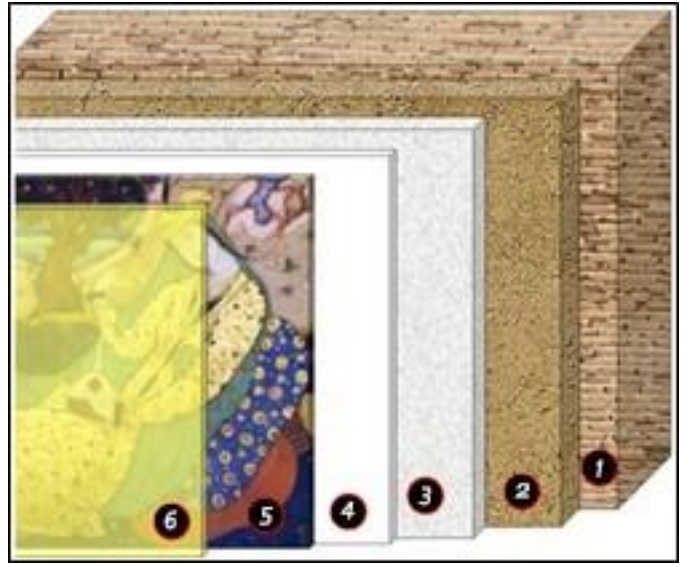

شكل (: طرح كلى از لايههاى يك ديوارنغاره: (. تكيه كاه ז. آستر

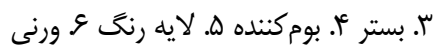

Fig; 1: Schematic picture of the layers of a wall paintings:

1. Support 2. Scratch coat 3. Fine coat 4.Primary layer 5. Paint layer 6. Varnish

عشرين و سبعمائه (VTD هـق.) نوشتهشده اسـت. ايـن بنـا داراى آرايههاى معمارى متعددى است كه ازجمله مىتوان إنهان

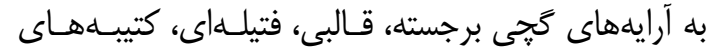

جهت اجراى لايئ آستر (لايئ رويى تكيهًاه و لايئ زيـرين

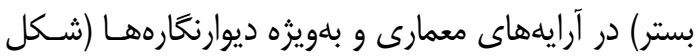

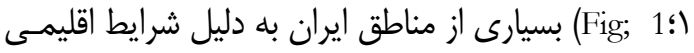

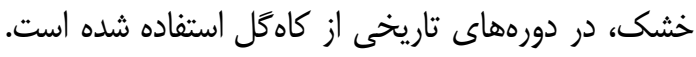

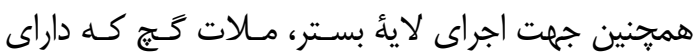

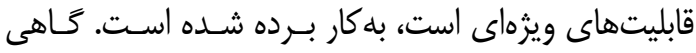

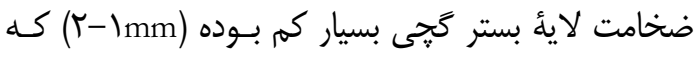

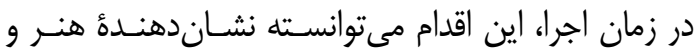

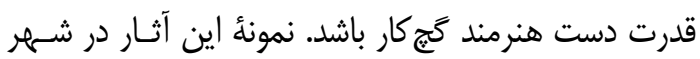

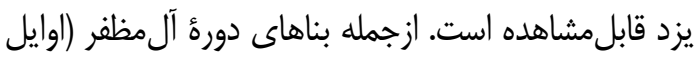

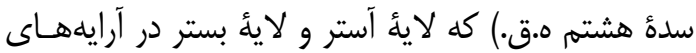

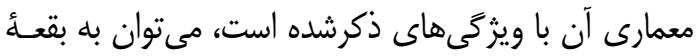

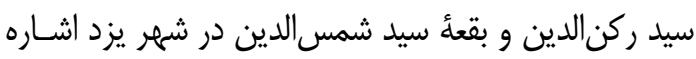
نمود. بقعأ سيد ركنالدين در زمان ساخت، كاربرى مدرسـهـ

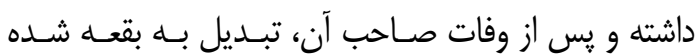

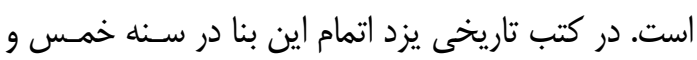

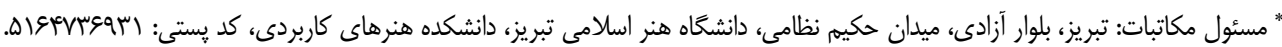

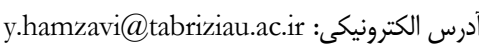
(C) جق نشر براي نويسنده(كان) بوده و نويسنده تحت مجوز Creative Commons Attribution License به مجله اين امكان را مي دهد كه مقاله קإٍ شده را با ديخران به اشتراك كذاشته منوط به اينكه حقوق مؤلف اثر حفظ شود و به انتشار اوليه مقاله در اين مجله اشاره شود. 


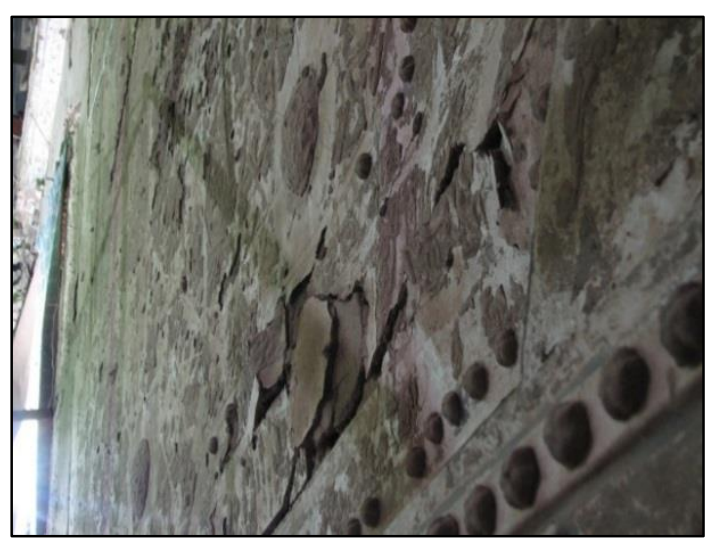

شكل ": فرسوده شدن و ازبين رفتن بخشهاى زيادى از لائُ آستر

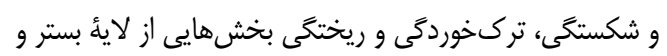
ضعف اتصال آرايههاى كَجى قالبى به زمينئ بستر

Fig; 3: Wearing out and destroying large parts of the Scratch coat and fracture, crackling and casting parts of the Fine coat and the weakness of bonding gypsum arrays to the ground

شدن اقدامات مرمتى شده و باعث ايجاد محـدوديتهـايى

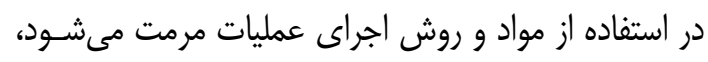
رنغ سفيد زمينه آرايـهـهـاى معمـارى در ايـن بناهاسـت.

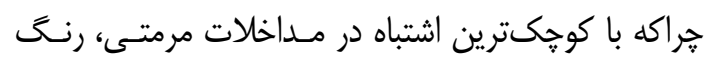

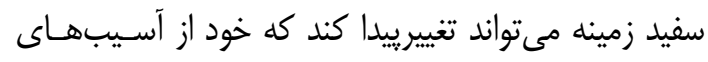

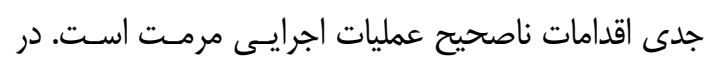

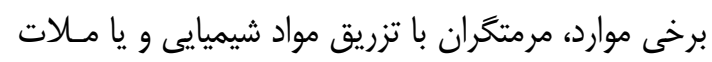

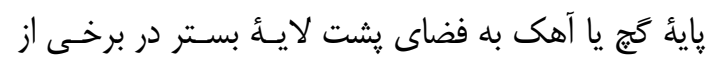

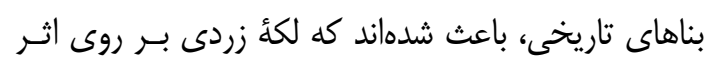

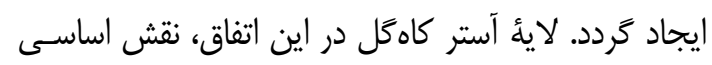

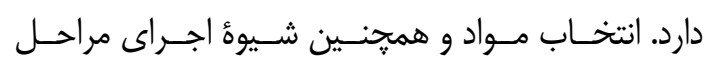
استحكامبخشى بسيار حائز اهميت است.

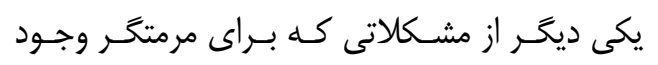

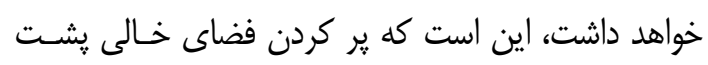

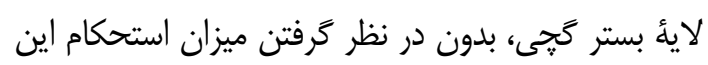

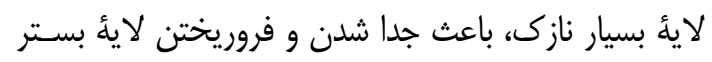

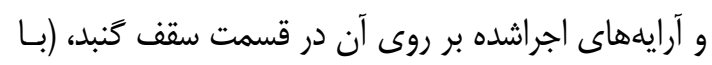

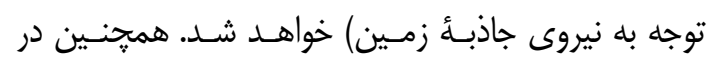

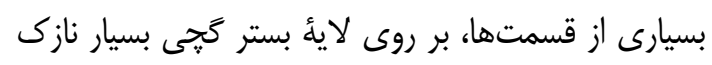

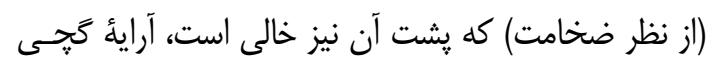

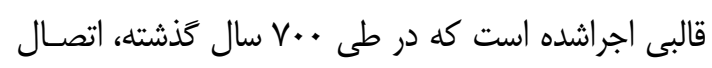

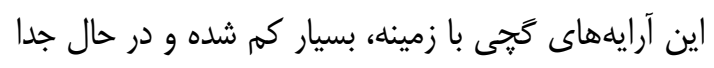

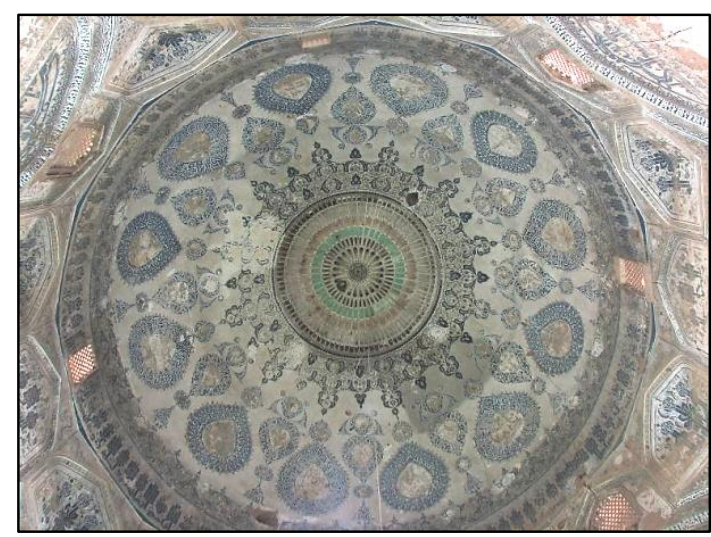

شكل ז: آرايههاى معمارى سقف كَنبد بقعهُ سيدركن الدين Fig; 2: Architectural arrays of dome roof of Rokn al-Din mausoleum

نقاشى، نقاشــهـاى تزيينسى، آرايـهـهـاى طلاكــارى و

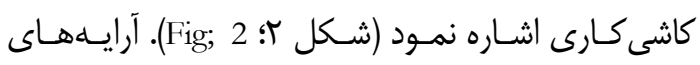
معمارى فضاى داخلى كنبدخانه از نظر وسعت، نسبتاً حجم أنما

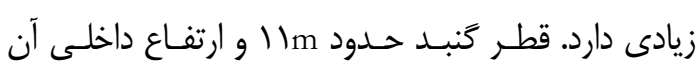

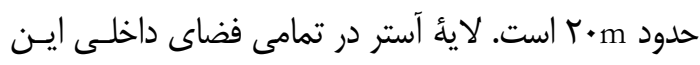

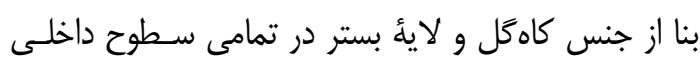

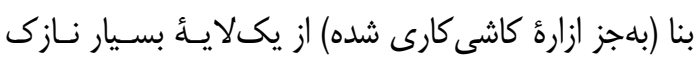

$$
\text { كَ است. }
$$

مسئله اساسى در رابطه با آرايههاى ايـن قبيـل ابنيـهـ زمانى رخ مىدهد كه كاه و الياف كَياهى موجود در كاهكل

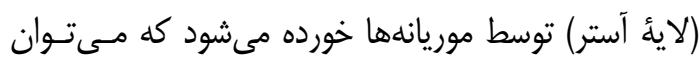

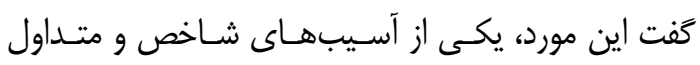

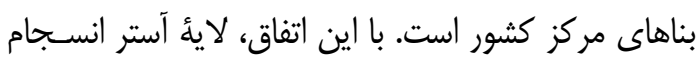

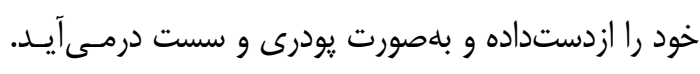

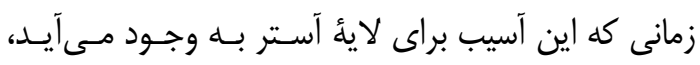

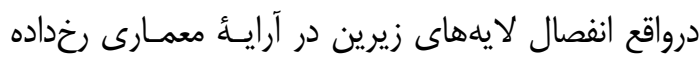

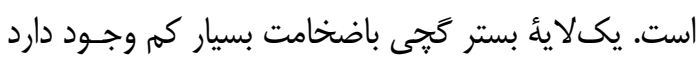

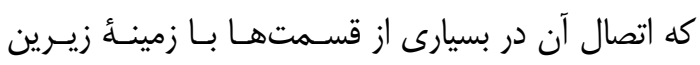

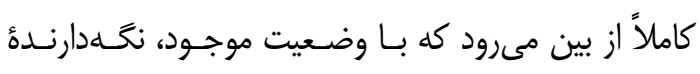

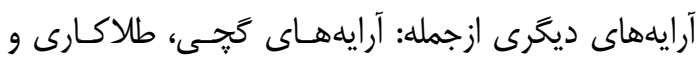

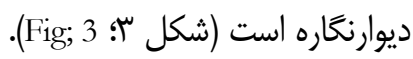
اقدامات حفاظتى جهت ماندكارتر كـردن آرايـهــاى

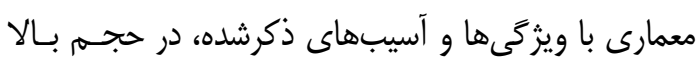

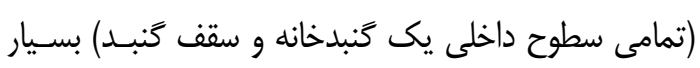

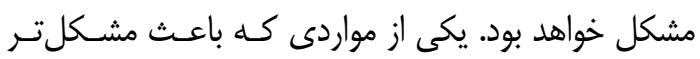


ركنالدين يزد، تصميم كرفته شد عمليات استحكامبخشى

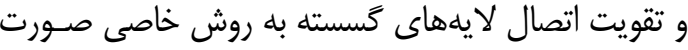

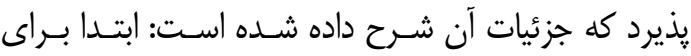

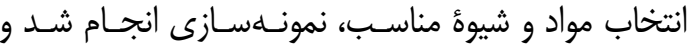

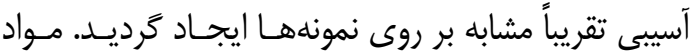
مختلفى كه در ذيل ارائه مـى كَـردد مـورد آزمـايش قـرار

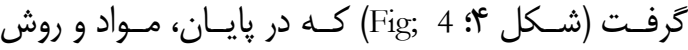
نوشتهشده در شمارة 19 انتخاب شد (جدول (ا؛ 1 (Table).

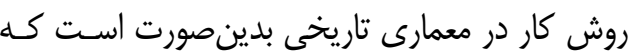

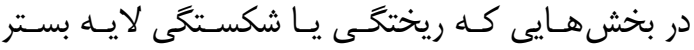

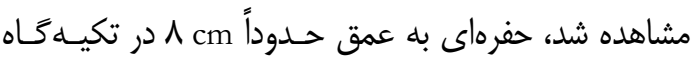
(ديوار يا سقف آجرى يا خشـتى) ايجـاد كَرديـد كـهـ قطـر

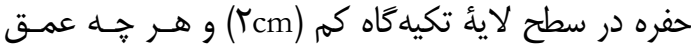

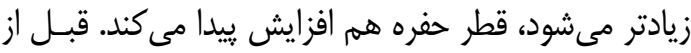

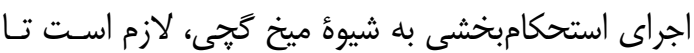

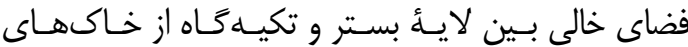
فرسوده پاك شود و سطح رويى و زيرين لائُ بستر تثبيت كردد (شكل ه؛ Fig; 5 (F). مرحلئ بعد، تزريق و اجراى ملات كج بــه لايسهــاى زيرين است. براى اين كار از گَج بومى منطقأ يزد و ويـودر سريش (جهت بالاتر بردن استحكام و همجنـين كنـدكير

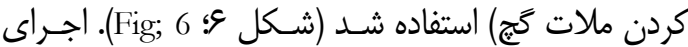

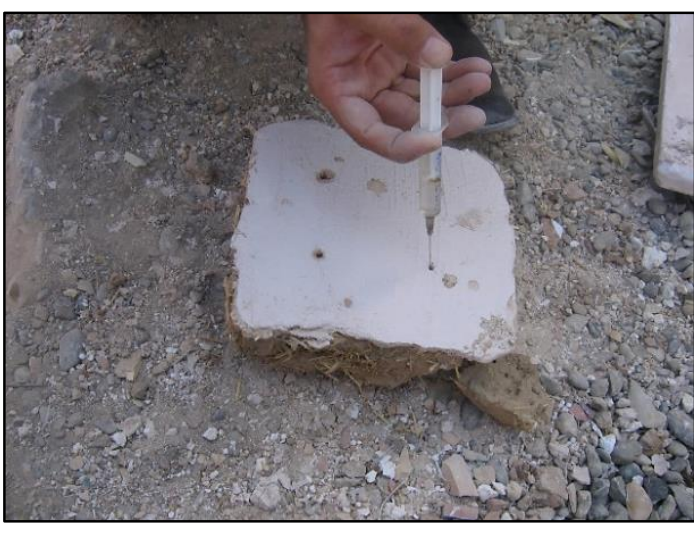
شكل f: نمونهسازى و شبيهسازى لايههاى تكيه كاه، آستر، بستر و تزريق مواد استحكامبخش بين لايهها

layers of Fig; 4: Sample construction and simulation support, Scratch coat, Fine coat and Injection of reinforcing materials between layers

شدن است (لائُ نازك گج كه مانند يوستأ تخممرغ بـوده

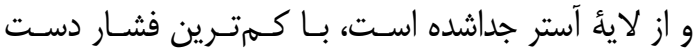

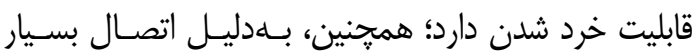

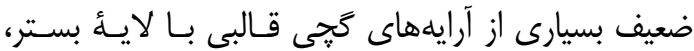

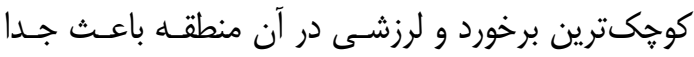

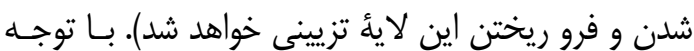

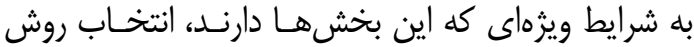
صحيح اجراى مراحل استحكامبخشى بسيار مهـهم خواهـــ

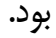
اين مرحله از حساسيت بـالايى برخـوردار اسـت. عمليـات

جدول (: مواد مورد استفاده جهت تثبيت و استحكامبخشى لايههاى بستر، آستر و تكيه كاه

Table 1: Materials for stabilization and consolidation of fine coat layer and scratch coat and support

\begin{tabular}{|c|c|c|c|c|c|c|c|c|c|c|c|c|c|c|c|c|c|c|c|}
\hline & 1 & 2 & 3 & 4 & 5 & 6 & 7 & 8 & 9 & 10 & 11 & 12 & 13 & 14 & 15 & 16 & 17 & 18 & 19 \\
\hline T & TA & TA & TB & TB & TC & TC & TC & TC & TC & TC & --- & --- & --- & TB & TA & TA & TA & --- & TA \\
\hline TP & --- & TA & --- & TB & --- & --- & --- & --- & TB & TC & --- & --- & --- & --- & --- & TC & --- & --- & TC \\
\hline M & ID & IA & IA & IA & IA & ID & IC & IB & IB & IA & IA & IB & ID & ID & IA & IA & IC & IC & IC \\
\hline
\end{tabular}

Table guide

T: Stabilization of wall paintings surface

TP: Stabilization of under the fine coat

M: Injection of prepared mortar on under the fine coat and a hole in the support

TA: solution of Acryloid B72 6\% in acetone

TB: solution of Acryloid $\mathrm{B}_{72} 3 \%$ in acetone

TC: solution of Acryloid $B_{72} 12 \%$ in acetone

IA: lime whitewash

IB: Gypsum mortar

IC: Gypsum mortar with Powder of the series

ID: Gypsum mortar with Plextol B 500
راهنماى جدول

T

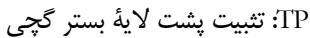

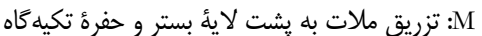

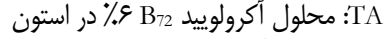

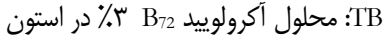

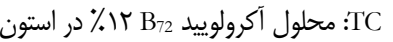
IA

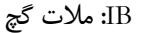
(IC : ملات گج و رزين يلكستول سريش :ID 


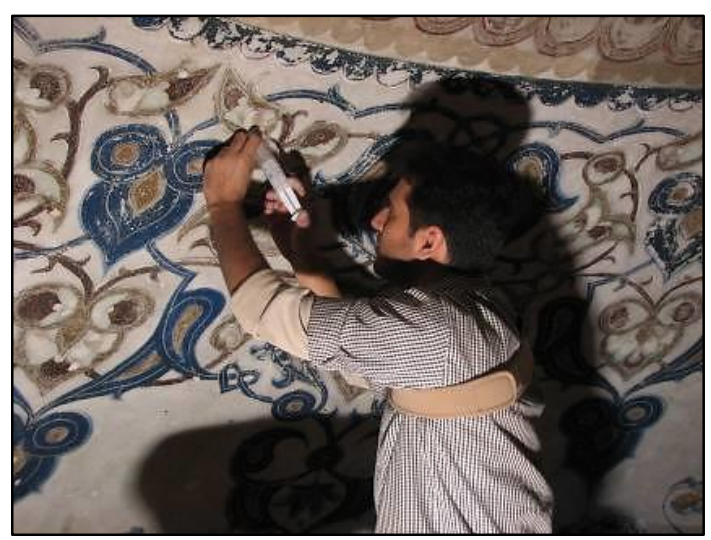

شكل \&: تزريق ملات در فضاهاى خالى بين لايهها و استحكامبخشى

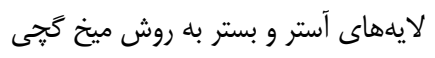

Fig; 6: Injection of mortar in void spaces between layers and strengthening of fine coat and scratch coat Gypsum Nail method

است و هميجنين اين ملات مانند يـك مـيخ، لايسه بسـتر

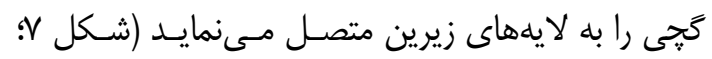
Fig; 7

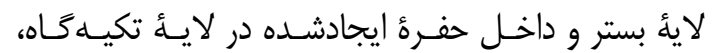

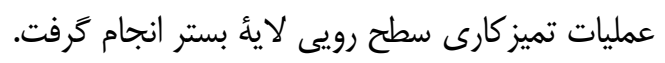

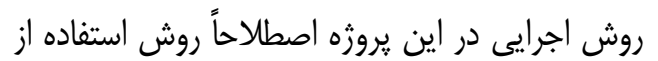

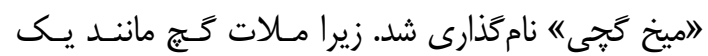

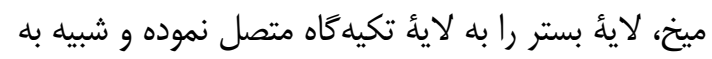
ميخ عمل مى كند. اجراى ميخهاى كَجى بــا فاصـلههـاى نسـبتاً معـين

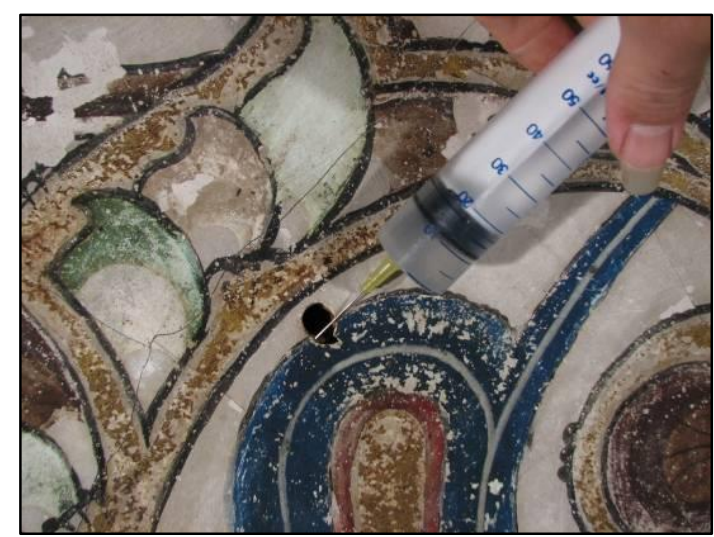

شكل ه: تثبيت پشت و روى لائُ بستر سقف كنبد بقعهُ سيد ركنالدين Fig; 5: Stabilization of back and forth of fine coat in dome roof of Rokn al-Din mausoleum

تزريق ملات كج به قسمت يشت لائَ بسـتر و همجنــين

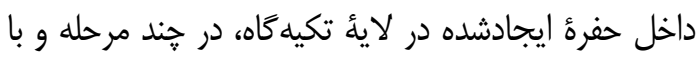

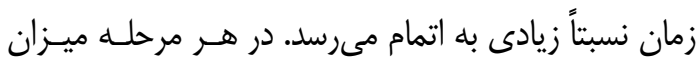

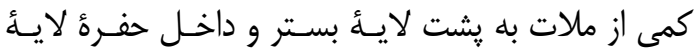

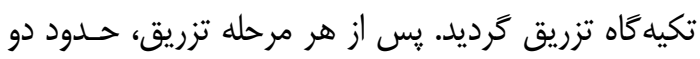

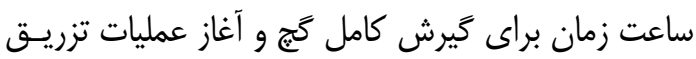
بعدى نياز است. در اين مدت، هيج اقـدامى نبايـد صـورت كاتِ

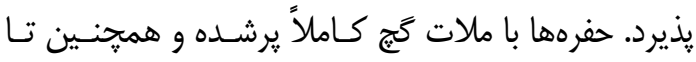

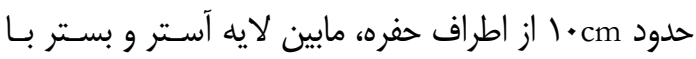

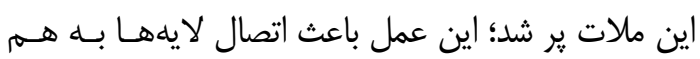

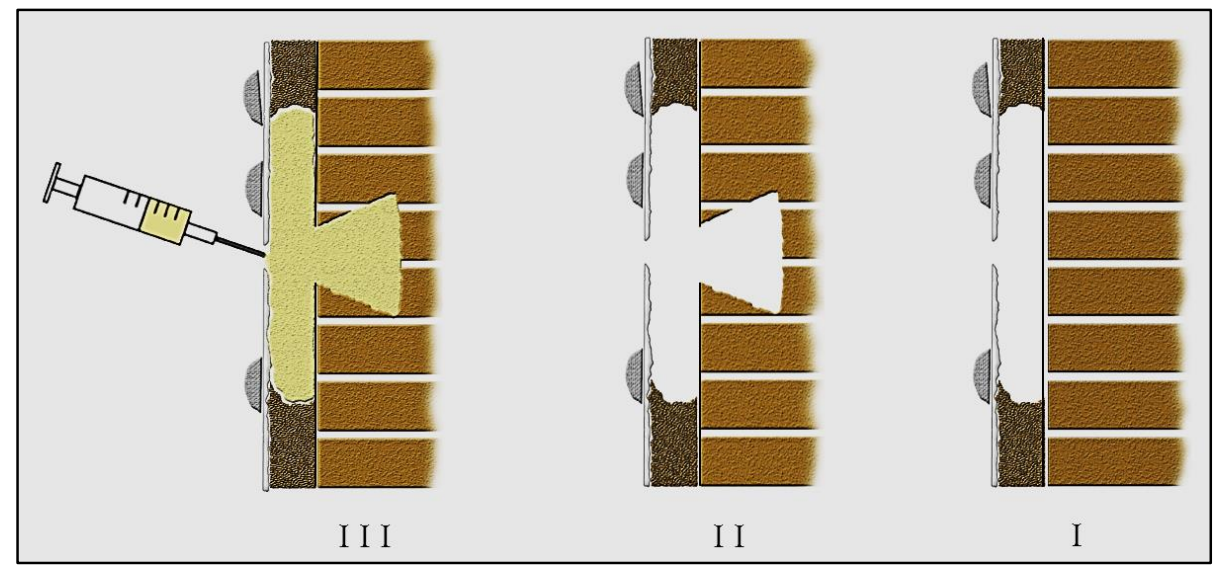

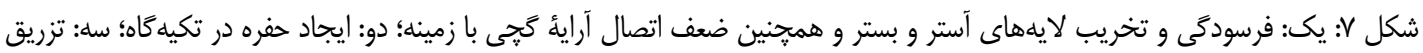

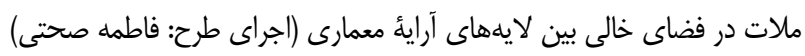

Fig; 7: I: Exhaustion and destruction of fine and scratch coat layers, as well as the weakness of the connection of the gypsum array with the field; II: Creating a hole in the support; III: Injection of mortar in void spaces between layers and strengthening of fine and scratch coat Gypsum Nail method 


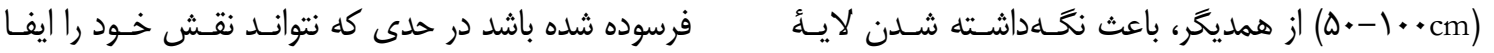

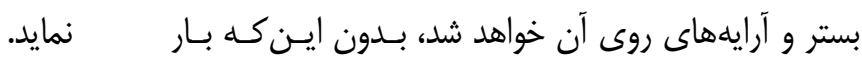

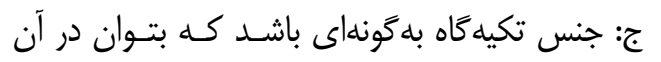

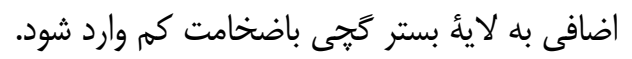

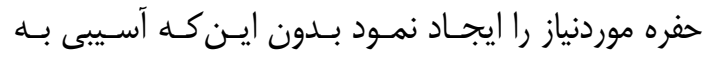

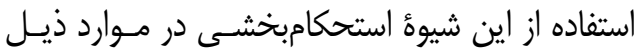
لايههاى مختلف آرايههاى معمارى برسد. مىتواند نقش مفيدى را ايفا نمايد:

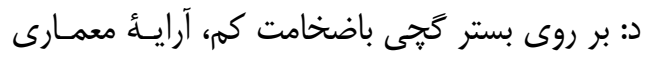
الف: ضخامت لائُ بستر در آرايههاى معمارى بسـيار

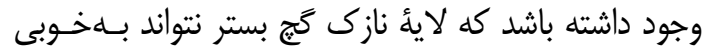
كم باشد (كمتر از ऍ ب: لائٔ آستر به هر دليلى از بين رفته باشد يا بسـيار وزن لايههاى رويى را تحمل كند. 